\title{
壳聚糖@铜催化吲哚与硫粉和芳基碘化物的 C3-硫醚化反应
}

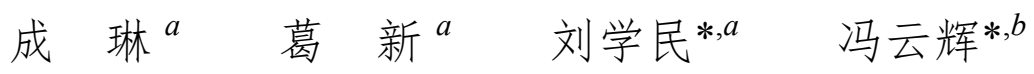 \\ ( ${ }^{a}$ 江南大学化学与材料工程学院 江苏无锡 214122) \\ ( ${ }^{b}$ 广州大学体育教育系 广州 510006)
}

\begin{abstract}
摘要 针对以硫单质为硫源的吲哚环中 $\mathrm{C}-\mathrm{S}$ 键构筑中催化剂无法回收的问题, 制备了 2-吡啶甲酸改性壳聚糖(PACS) 负载不同类型铜的催化剂，用于催化吲哚、硫粉和碘苯三组分反应一锅法制备 C-3 位硫醚基吲哚，反应收率高达 $92 \%$, 底物适用性良好. 对催化效果最佳的催化剂(PACS@ $\left.\mathrm{Cu}(\mathrm{OAc})_{2}\right)$ 进行了热重分析(TGA)、扫描电子显微镜 $(\mathrm{SEM}) 、 \mathrm{X}$ 射线 光电子能谱(XPS)等表征与分析, 结果表明该催化剂具有不需额外添加配体、易于分离回收及可重复使用的优点. 关键词 壳聚糖; 吲哚; 硫粉; 催化; 非均相
\end{abstract}

\section{Chitosan@Cu-Catalyzed C3-Sulfenylation of Indoles with Sulfur Powder and Aryl lodides}

\author{
Cheng, $\operatorname{Lin}^{a} \quad$ Ge, Xin ${ }^{a} \quad$ Liu, Xuemin ${ }^{*, a} \quad$ Feng, Yunhui ${ }^{*, b}$ \\ ( ${ }^{a}$ School of Chemical and Material Engineering, Jiangnan University, Wuxi, Jiangsu 214122) \\ ( ${ }^{b}$ Department of Physical Education, Guangzhou University, Guangzhou 510006)
}

\begin{abstract}
Aiming at the problem that the catalyst can not be recovered during the construction of the $\mathrm{C}-\mathrm{S}$ bond in the indole ring with sulfur powder as the sulfur source, 2-pyridinecarboxylic acid modified chitosan (PACS) catalyst with different type of copper was prepared, which was used to catalyze the three-component reaction of indole, sulfur powder and iodobenzene to prepare C-3 thioether-based indole in a one-pot method. The reaction yield is as high as $92 \%$, and the substrate has good applicability. The most suitable catalyst $\left[\mathrm{PACS} @ \mathrm{Cu}(\mathrm{OAc})_{2}\right]$ was characterized and analyzed by thermogravimetric analysis (TGA), scanning electron microscope(SEM), X-ray photoelectron spectroscopy(XPS), etc. It shows that the catalyst has the advantages of no additional ligand, easy separation and reusable.

Keywords chitosan; indole; sulfur powder; catalyze; heterogeneous
\end{abstract}

$\mathrm{C}-\mathrm{S}$ 键的构建是有机合成中重要的一部分, 已广 泛应用在材料、药物化学、农药及染料化学等领域 ${ }^{[1]}$. 其 中, 吲哚类含硫化合物及其衍生物作为一类重要的含硫 化合物, 在抗菌 ${ }^{[2]}$ 、治疗心血管疾病 ${ }^{[3]}$ 和癌症 ${ }^{[4]}$ 、抗人类 免疫缺陷病毒 $(\mathrm{HIV})^{[5]}$ 等生物医药领域 ${ }^{[6]}$ 的应用效果显 著. 因此研究吲哚类含硫化合物 $\mathrm{C}-\mathrm{S}$ 键的构筑具有重 要意义 ${ }^{[7]}$. 传统用于合成吲哚含硫衍生物的硫源试剂, 以硫梄 ${ }^{[8]}$ 、硫醇 ${ }^{[9]}$ 、砜 ${ }^{[10]}$ 、硫氰酸盐 ${ }^{[11]}$ 等为代表, 主要 面临剧毒和恶臭问题, 同时硫原子的孤对电子强配位性 很容易和活性中心形成牢固的络合物, 使得催化剂失 活 ${ }^{[12]}$. 此外, 硫醇也易与二硫代化合物发生氧化偶联等 副反应 ${ }^{[13]}$. 因而清洁的硫源用于吲哚的含硫衍生物的
合成具有显著的优势.

近年来, 硫粉 $\left(\mathrm{S}_{8}\right)$ 因其廉价易得且在 $\mathrm{C}-\mathrm{S}$ 键的构筑 中具有较高的原子经济性等优势而倍受关注 ${ }^{[14]}$. 通常, 具有离去基团 $\left[\right.$ 如 $\mathrm{Br} 、 \mathrm{I}$ 或 $\mathrm{B}(\mathrm{OH})_{2}$ 的底物可在过渡金属 催化下与元素硫进行偶联 ${ }^{[15]} .2014$ 年, Shibahara 等 ${ }^{[16]}$ 将 硫元素通过亲电取代, 以原位生成 $\mathrm{S}^{2+}$ 的形式实现芳香 杂环化合物的直接 $\mathrm{C}-\mathrm{H}$ 键硫化生成二芳基硫化物. $\mathrm{Li}$ 等 ${ }^{[17]}$ 在 2016 年使用 $\mathrm{Pd}(\mathrm{OAc})_{2}$ 来催化吲哚与芳基硼酸和 $\mathrm{S}_{8}$ 进行反应, 实现吲哚环上的硫醚化, 具有很好的底物 适用性. 同年, Ravi 等 ${ }^{[14 \mathrm{~d}]}$ 报道了铜催化下使用硫粉和芳 基卤化物一锅法区域选择性地合成咪唑并杂环化合物, 该反应体系可提供高收率的硫代芳基咪唑并杂环化合

* Corresponding authors. E-mail: 1xm@jiangnan.edu.cn; 13795553266@163.com Received January 23, 2020; revised April 29, 2020; published online May 7, 2020. Project supported by the National Natural Science Foundation of China (No. 21606104). 国家自然科学基金(No. 21606104)资助项目. 
物, 具有良好的官能团耐受性. 2019 年, 本课题组 ${ }^{[18]}$ 以 单质硫为硫源, 开发了铜催化下的吲哚 C-3 位硫醚化反 应体系. 众所周知, 硫粉相对于其他含硫化合物来说具 有一定的惰性 ${ }^{[19]}$, 因此常常需要添加大量金属催化剂 和额外配体等较为苛刻的反应条件才可顺利参与有机 反应，这大大限制了硫粉在有机反应中的应用.

以单质硫为硫源的 $\mathrm{C}-\mathrm{S}$ 偶联反应体系的研究中所 用催化剂多为均相催化剂, 该类催化剂以分子或者离子 的形态与反应物发生相互作用, 活性中心均一, 具有高 活性和高选择性, 但是回收分离较为困难. 相对而言, 非均相催化剂分离更为简单, 易于实现化工的连续化过 程 ${ }^{[20]}$. 因此, 开发以单质硫为硫源的吲哚硫醚化反应的 非均相催化剂开发, 对于实现催化剂的回收和绿色化工 过程具有重要意义. 目前, 在吲哚环的功能化反应中非 均相催化剂已得到成功应用. 如 Krishnan 等 ${ }^{[21]}$ 报道了一 种利用石墨碳氮化物纳米复合材料负载金属, 在室温下 吲哚 C-3 位上的烯烃化合物的合成. Hong 等 ${ }^{[22]}$ 通过制备 一种以沸石为载体的钯催化剂, 实现了高效催化吲哚 C-2 位衍生物的合成, 且该催化剂表现出较好的可回用 性能. Shimizu 等 ${ }^{[23]}$ 报道了一类易于回收利用的 $\mathrm{Pt} / \gamma-$ $\mathrm{Al}_{2} \mathrm{O}_{3}$ 纳米簇催化剂, 能有效促进吲哚 C-3 位上的烷基 化反应. 载体是非均相催化剂的重要组成部分, 目前以 分子篎和碳基为主要代表. 作为来源广泛、可生物降解 的多糖类化合物, 壳聚糖 $(\mathrm{CS})$ 具有大量易修饰基团, 并 可通过改性增强其螯合金属的能力 ${ }^{[24]}$, 且因其难溶于 大部分有机溶剂 ${ }^{[25]}$, 是非均相催化剂的理想绿色碳基 载体 ${ }^{[26]}$.

本工作组设计了一种高效可回收的 2-吡啶甲酸改 性壳聚糖(PACS)负载铜配合物的催化剂, 用于吲哚、硫 粉和碘苯三组分体系一锅法合成 C-3 位硫醚基吲哚的反 应(如 Scheme 1 所示), 并对其催化活性、稳定性进行了 评价与测试. 该催化剂易于回收利用, 具有良好的循环 使用性能, 且反应体系无需额外添加配体, 更加经济简 单.

\section{1 结果与讨论}

\section{1 反应条件优化}

本工作使用碘苯、硫粉和 $N$-甲基吲哚三组分一锅法 制备 C-3 位硫醚基吲哚的反应为模型反应, 考察不同反 应条件对催化效果的影响(表 1). 首先对催化剂进行了 笁选(表 1, Entries 1 7), 结果表明 PACS@Cu(OAc) 2 的 活性最高, 与未改性的壳聚糖铜配合物相比, 对壳聚糖 骨架进行修饰后提高了配合物的催化活性. 作为反应的 缚酸剂, 选择合适的碱也会有利于反应的正向进行 ${ }^{[27]}$, 因此对不同种类的碱进行笁选(表 1, Entries 6, 8 14),
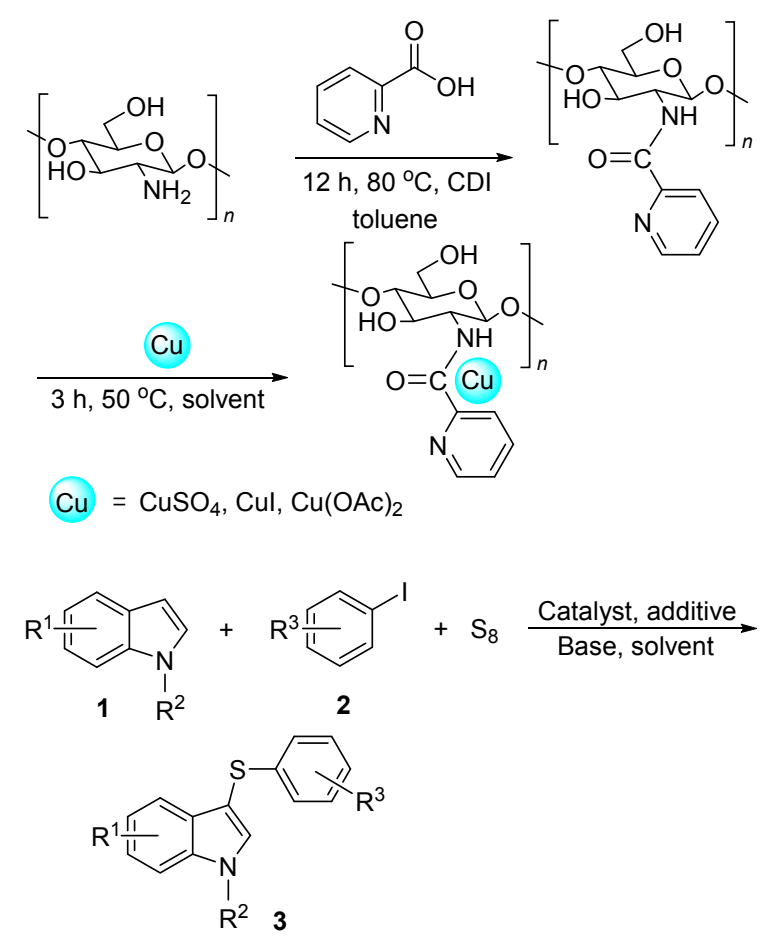

图式 1 PACS $@ \mathrm{Cu}(\mathrm{OAc})_{2}$ 催化剂的制备及应用 Scheme 1 Preparation and application of PACS@ $\mathrm{Cu}(\mathrm{OAc})_{2}$ catalyst

发现 $\mathrm{Cs}_{2} \mathrm{CO}_{3}$ 的效果优于其他碱. 当反应使用强碱 $\mathrm{NaOH}$ 时, 收率为 0 (表 1, Entry 11). 这可能是因为在强碱条件 下碘苯与硫生成副产物二苯硫醚，导致反应无法进行. 随后对反应体系中的含碘添加剂进行篮选(表 1, Entries $10,15 \sim 17)$, 发现 $\mathrm{KI}$ 为最佳含碘添加剂. 此外, 对不同 溶剂进行篎选(表 1, Entries 10,18２1), 结果表明 $N, N$ 二甲基甲酰胺(DMF)最有利于该反应进行. 还考察了温 度对反应的影响(表 1, Entries 22 24), 发现降低温度后 产物的收率明显降低, 而升高温度并不能使收率进一步 提高. 最后, 进行了浸出实验验证催化剂的非均相性 (表 1, Entry 25). 当反应进行 $6 \mathrm{~h}$ 且收率达到 $60 \%$ 时, 将 $\mathrm{PACS} @ \mathrm{Cu}(\mathrm{OAc})_{2}$ 固体从反应体系中分离出来, 随后继 续反应 $6 \mathrm{~h}$ 发现收率并未上升. 这表明铜盐在载体表面 是稳定的, 且催化剂确实为非均相催化剂. 因此, 该反 应的最佳反应条件为: $\mathrm{PACS} @ \mathrm{Cu}(\mathrm{OAc})_{2}$ 为催化剂, $\mathrm{Cs}_{2} \mathrm{CO}_{3}$ 为碱, $\mathrm{KI}$ 为添加剂, $\mathrm{DMF}$ 为溶剂, $110{ }^{\circ} \mathrm{C}$, 且无 需额外再添加配体即可实现反应.

\section{2 催化剂的表征}

\section{2 .1 催化剂的红外分析(FT-IR)}

首先从分子结构的官能团方面分析壳聚糖 $(\mathrm{CS}) 、 2-$ 吡啶甲酸改性壳聚糖(PACS)和 2-吡啶甲酸改性壳聚糖 铜配合物(PACS@ $\left.\mathrm{Cu}(\mathrm{OAc})_{2}\right)$ 三种材料之间分子结构的 变化. 采用FT-IR 对 CS, PACS 及 PACS@Cu(OAc) $)_{2}$ 进行 
表 1 反应条件优化 ${ }^{a}$

Table 1 Optimization of reaction conditions

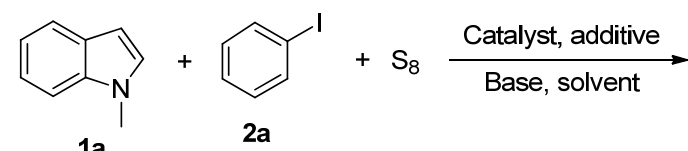

$1 a$<smiles>Cn1cc(Sc2ccccc2)c2ccccc21</smiles>

3a

\begin{tabular}{|c|c|c|c|c|c|}
\hline Entry & Catalyst & Base & Additive & Solvent & $\begin{array}{c}\text { Yield }^{b} / \\
\%\end{array}$ \\
\hline 1 & $\mathrm{CS} @ \mathrm{Cu}(\mathrm{OAc})_{2}$ & $\mathrm{NaOAc}$ & KI & DMSO & Trace \\
\hline 2 & $\mathrm{CS} @ \mathrm{CuSO}_{4}$ & $\mathrm{NaOAc}$ & $\mathrm{KI}$ & DMSO & Trace \\
\hline 3 & CS@CuI & $\mathrm{NaOAc}$ & $\mathrm{KI}$ & DMSO & Trace \\
\hline 4 & $\mathrm{Cu}(\mathrm{OAc})_{2}$ & $\mathrm{NaOAc}$ & KI & DMSO & 0 \\
\hline 5 & PACS@Cu(OAc) $)_{2}$ & $\mathrm{NaOAc}$ & KI & DMSO & 50 \\
\hline 6 & PACS@ $\mathrm{CuSO}_{4}$ & $\mathrm{NaOAc}$ & $\mathrm{KI}$ & DMSO & 5 \\
\hline 7 & PACS@CuI & $\mathrm{NaOAc}$ & KI & DMSO & 10 \\
\hline 8 & $\mathrm{PACS} @ \mathrm{Cu}(\mathrm{OAc})_{2}$ & $\mathrm{Na}_{2} \mathrm{CO}_{3}$ & KI & DMSO & 40 \\
\hline 9 & $\mathrm{PACS} @ \mathrm{Cu}(\mathrm{OAc})_{2}$ & $\mathrm{~K}_{2} \mathrm{CO}_{3}$ & $\mathrm{KI}$ & DMSO & 12 \\
\hline 10 & $\mathrm{PACS} @ \mathrm{Cu}(\mathrm{OAc})_{2}$ & $\mathrm{Cs}_{2} \mathrm{CO}_{3}$ & KI & DMSO & 65 \\
\hline 11 & PACS@Cu(OAc) $)_{2}$ & $\mathrm{NaOH}$ & KI & DMSO & 0 \\
\hline 12 & $\mathrm{PACS} @ \mathrm{Cu}(\mathrm{OAc})_{2}$ & $\mathrm{NaHCO}_{3}$ & $\mathrm{KI}$ & DMSO & 50 \\
\hline 13 & $\mathrm{PACS} @ \mathrm{Cu}(\mathrm{OAc})_{2}$ & $\mathrm{Et}_{3} \mathrm{~N}$ & KI & DMSO & 15 \\
\hline 14 & PACS@Cu(OAc) $)_{2}$ & - & $\mathrm{KI}$ & DMSO & 0 \\
\hline 15 & $\mathrm{PACS} @ \mathrm{Cu}(\mathrm{OAc})_{2}$ & $\mathrm{Cs}_{2} \mathrm{CO}_{3}$ & $\mathrm{NH}_{4} \mathrm{I}$ & DMSO & 55 \\
\hline 16 & $\mathrm{PACS} @ \mathrm{Cu}(\mathrm{OAc})_{2}$ & $\mathrm{Cs}_{2} \mathrm{CO}_{3}$ & $\mathrm{I}_{2}$ & DMSO & 45 \\
\hline 17 & $\mathrm{PACS} @ \mathrm{Cu}(\mathrm{OAc})_{2}$ & $\mathrm{Cs}_{2} \mathrm{CO}_{3}$ & - & DMSO & 0 \\
\hline 18 & PACS@Cu(OAc) $)_{2}$ & $\mathrm{Cs}_{2} \mathrm{CO}_{3}$ & KI & $\mathrm{CH}_{3} \mathrm{CN}$ & 0 \\
\hline 19 & $\mathrm{PACS} @ \mathrm{Cu}(\mathrm{OAc})_{2}$ & $\mathrm{Cs}_{2} \mathrm{CO}_{3}$ & KI & DMF & 88 \\
\hline 20 & $\mathrm{PACS} @ \mathrm{Cu}(\mathrm{OAc})_{2}$ & $\mathrm{Cs}_{2} \mathrm{CO}_{3}$ & KI & Toluene & 0 \\
\hline 21 & $\mathrm{PACS} @ \mathrm{Cu}(\mathrm{OAc})_{2}$ & $\mathrm{Cs}_{2} \mathrm{CO}_{3}$ & $\mathrm{KI}$ & 1,4-Dioxane & 42 \\
\hline 22 & PACS@Cu(OAc) & $\mathrm{Cs}_{2} \mathrm{CO}_{3}$ & KI & DMF & $53^{c}$ \\
\hline 23 & $\mathrm{PACS} @ \mathrm{Cu}(\mathrm{OAc})_{2}$ & $\mathrm{Cs}_{2} \mathrm{CO}_{3}$ & $\mathrm{KI}$ & DMF & $75^{d}$ \\
\hline 24 & $\mathrm{PACS} @ \mathrm{Cu}(\mathrm{OAc})_{2}$ & $\mathrm{Cs}_{2} \mathrm{CO}_{3}$ & $\mathrm{KI}$ & DMF & $88^{e}$ \\
\hline 25 & $\mathrm{PACS} @ \mathrm{Cu}(\mathrm{OAc})_{2}$ & $\mathrm{Cs}_{2} \mathrm{CO}_{3}$ & $\mathrm{KI}$ & DMF & $60^{f}$ \\
\hline
\end{tabular}

${ }^{a}$ Reaction conditions: 1a $(1.0 \mathrm{mmol}), \mathbf{2 a}(2 \mathrm{mmol}), \mathrm{S}_{8}(2 \mathrm{mmol})$, catalyst $(100$ $\mathrm{mg})$, additive $(10 \mathrm{mmol} \%)$, base $(1.0 \mathrm{mmol})$, solvent $(5 \mathrm{~mL}), 12 \mathrm{~h}, 110{ }^{\circ} \mathrm{C}$. ${ }^{b}$ Isolated yield. ${ }^{c}$ Under $90{ }^{\circ} \mathrm{C} .{ }^{d}$ Under $100{ }^{\circ} \mathrm{C} .{ }^{e}$ Under $120{ }^{\circ} \mathrm{C} .{ }^{f}$ Leaching test of the model reaction conducted under optimized conditions for $6 \mathrm{~h}$.

表征(图 1). 曲线 a 中 $\mathrm{CS}$ 的特征吸收峰出现在 3427 $(\mathrm{O}-\mathrm{H}$ 伸缩振动峰 $) 、 2906(\mathrm{C}-\mathrm{H}$ 伸缩振动峰 $) 、 1590$ $(\mathrm{N}-\mathrm{H}$ 弯曲振动峰 $) 、 1378(\mathrm{C}-\mathrm{H}$ 弯曲振动峰 $) 、 1252$ (C-O-C 伸缩振动峰) 和 $1094(\mathrm{C}-\mathrm{O}$ 伸缩振动峰) $\mathrm{cm}^{-1}$. 与 $\mathrm{CS}$ 的 FT-IR 光谱相比, 曲线 $\mathrm{b}$ 中 PACS 在 1737 $\mathrm{cm}^{-1}$ 处出现新峰, 这是由 $\mathrm{C}=\mathrm{O}$ 的伸缩振动所致. 另外, 在 1526 和 $1284 \mathrm{~cm}^{-1}$ 处新出现了仲酰胺的 $\mathrm{C}-\mathrm{N}$ 伸缩振 动与 $\mathrm{N}-\mathrm{H}$ 的弯曲振动峰, 在 1471 和 $748 \mathrm{~cm}^{-1}$ 处出现 的新峰分别是吡啶环骨架的弯曲振动和伸缩振动所致. 这些变化表明, CS 已成功被改性并带有吡啶和羰基结
构. 在 FT-IR 谱图中 $\mathrm{b}$ 和 $\mathrm{c}$ 也出现差异, $1737 \mathrm{~cm}^{-1}$ 移动 到 $1741 \mathrm{~cm}^{-1}$, 这是由酰胺结构与铜的协调作用所致.

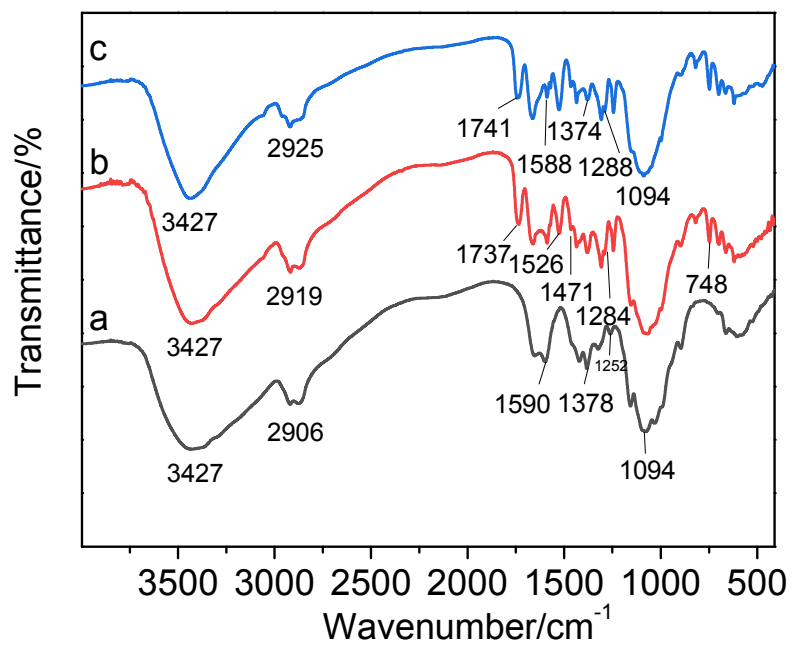

图 $1 \mathrm{CS}(\mathrm{a}), \mathrm{PACS}(\mathrm{b})$ 及 PACS@Cu(OAc) 2 (c)的红外谱图 Figure 1 FT-IR spectra of CS (a), PACS (b) and PACS@Cu$(\mathrm{OAc})_{2}(\mathrm{c})$

\subsection{2 催化剂的热重分析(TGA)}

壳聚糖、2-吡啶甲酸改性壳聚糖及其醋酸铜配合物 在氮气中由 $50{ }^{\circ} \mathrm{C}$ 升温至 $800{ }^{\circ} \mathrm{C}$ 的热重变化如图 2. 样 品随着程序升温有两处发生明显的质量损失 ${ }^{[28]}$ : 在 $50 \sim 100{ }^{\circ} \mathrm{C}$ 的升温过程中, 三种材料均出现轻微的质量 减少，该过程的质量损失主要是壳聚糖粉末中的水分蒸 发所致; 而在温度由 $250{ }^{\circ} \mathrm{C}$ 升温至 $350{ }^{\circ} \mathrm{C}$ 的过程中, 三 种材料出现约 $50 \%$ 的质量损失, 这是壳聚糖链发生断裂 所致。该现象表明，在低于 $150{ }^{\circ} \mathrm{C}$ 的温度下, PACS@ $\mathrm{Cu}(\mathrm{OAc})_{2}$ 仅蒸发掉了部分水而其主链并未被破坏. 由 此可知壳聚糖在 $110{ }^{\circ} \mathrm{C}$ 时依然可以保持结构的稳定, 这使得壳聚糖可以实现多次回收再利用.

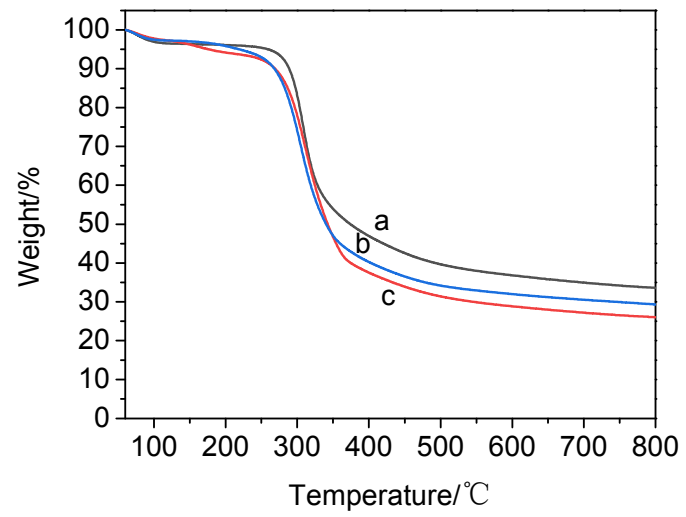

图 $2 \mathrm{CS}(\mathrm{a}), \mathrm{PACS} @ \mathrm{Cu}(\mathrm{OAc})_{2}$ (b)以及 PACS (c)的热重分析 Figure 2 TGA of CS (a), PACS $@ \mathrm{Cu}(\mathrm{OAc})_{2}$ (b) and PACS (c)

\subsection{3 催化剂的 X 射线衍射分析 (XRD)}

图 3 为 $\mathrm{CS}, \mathrm{PACS}$ 以及 $\mathrm{PACS} @ \mathrm{Cu}(\mathrm{OAc})_{2}$ 的 XRD 谱 
图. 在图 3a 中 $10.5^{\circ}$ 与 $20.3^{\circ}$ 位置的两个峰是壳聚糖的主 要特征峰 ${ }^{[29]}$. 而图 $3 b$ 显示 $10.5^{\circ}$ 处的峰减弱, 且 $20.3^{\circ}$ 处 的峰变得尖锐, 可能是由于羰基结构的形成和游离氨基 的减少所致. 由图 $3 \mathrm{c}$ 可以看出, 改性壳聚糖与铜螯合 后, $10.5^{\circ}$ 处的峰消失, 而 $13.6^{\circ}$ 和 $26.9^{\circ}$ 处的衍射峰出现, 推测发生该现象的原因是鳌合铜后分子结构中的共价 键发生变化, 导致其晶型变化. 此外, 图 3c 中并未出现 铜盐的峰，说明铜盐是均匀分布在载体上的 ${ }^{[30]}$.

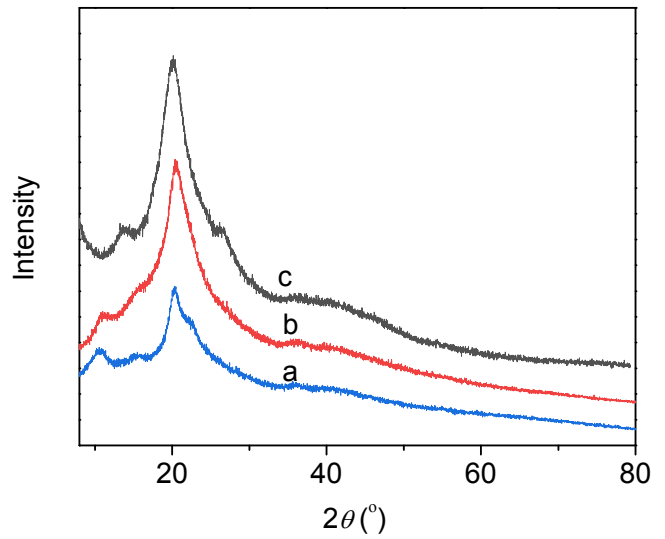

图 $3 \mathrm{CS}(\mathrm{a}), \mathrm{PACS}(\mathrm{b})$ 以及 PACS@Cu(OAc) $)_{2}$ (c) 的 XRD 谱图 Figure 3 XRD patterns of CS (a), PACS (b) and PACS@Cu$(\mathrm{OAc})_{2}(\mathrm{c})$

\subsection{4 催化剂的形貌观察(SEM)与 $\mathrm{X}$ 射线能谱分析 (EDS)}

如图 4 所示, 用扫描电镜记录了 CS 和 PACS 的不 同形貌. 与 CS 相对光滑的表面相比, 被改性后得到的 PACS 的表面有更多更深的褶皱, 这有利于壳聚糖与更 多的铜进行接触. 此外, 图 4c 和 $4 \mathrm{~d}$ 分别为 PACS 螯合 $\mathrm{Cu}(\mathrm{OAc})_{2}$ 后的样品及其使用两次后的形貌. 且结合后 续 EDS 分析可知在经过两次使用后, 催化剂上仍有铜 盐的存在.

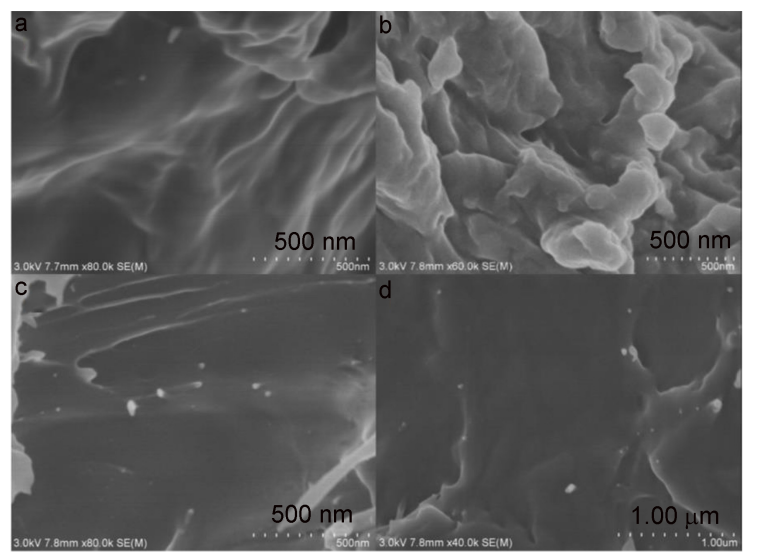

图 $4 \mathrm{CS}(\mathrm{a}), \mathrm{PACS}(\mathrm{b}), \mathrm{PACS} @ \mathrm{Cu}(\mathrm{OAc})_{2}$ (c)及使用两次后的 PACS@Cu(OAc $)_{2}(\mathrm{~d})$ 的 SEM 图

Figure 4 SEM images of CS (a), PACS (b), PACS@ $\mathrm{Cu}(\mathrm{OAc})_{2}$ (c) and recovered PCCS@(OAc) $)_{2}$ after the second run (d)
EDS 分析再次证实了 PACS $@ \mathrm{Cu}(\mathrm{OAc})_{2}$ 材料中铜的 存在. 从图 $5 \mathrm{~d}$ 可看出, 催化剂经过回收利用两次, 其负 载的铜依然存在, 经过铜含量测定可知, 未使用的 $\mathrm{PACS} @ \mathrm{Cu}(\mathrm{OAc})_{2}$ 材料中铜含量约为 $0.164 \mathrm{mmol} / \mathrm{g}$, 使 用两次后的材料中铜含量有所降低, 为 $0.148 \mathrm{mmol} / \mathrm{g}$.
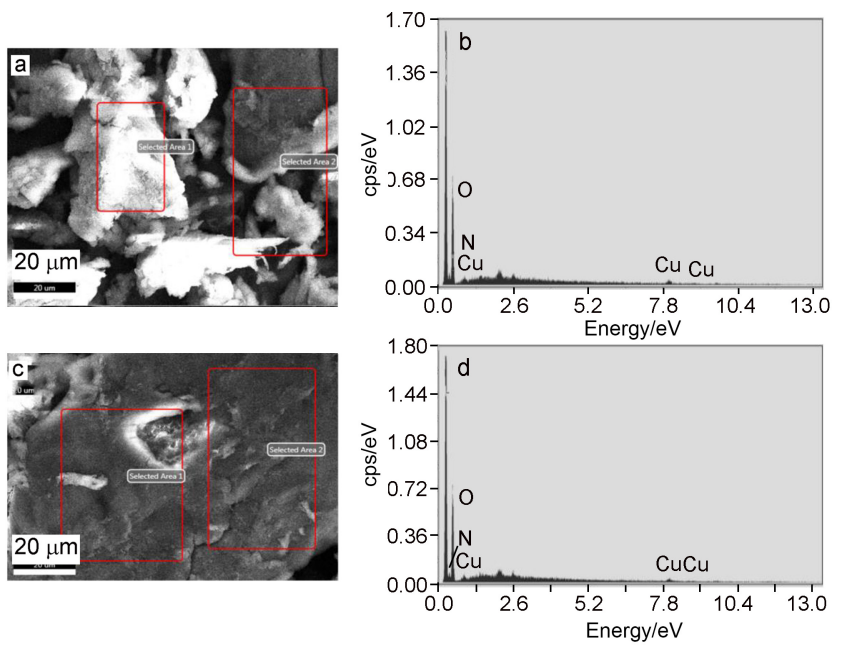

图 5 PACS@ $\mathrm{Cu}(\mathrm{OAc})_{2}(\mathrm{a}, \mathrm{b})$ 及使用两次后的 $\mathrm{PACS} @ \mathrm{Cu}-$ $(\mathrm{OAc})_{2}(\mathrm{c}, \mathrm{d})$ 的 EDS 图

Figure 5 EDS images of PACS $@ \mathrm{Cu}(\mathrm{OAc})_{2}(\mathrm{a}, \mathrm{b})$ and recovered PCCS@Cu(OAc) $)_{2}$ after the second run $(\mathrm{c}, \mathrm{d})$

\subsection{5 催化剂的 X 射线光电子能谱分析(XPS)}

图6a为 PACS@Cu(OAc) $)_{2}$ 与 PACS 的 XPS 全谱, 通 过对二者进行对比可以发现, 前者出现两个新特征峰, 即在 933.6 和 $953.4 \mathrm{eV}$ 位置出现的分别是 $\mathrm{Cu} 2 \mathrm{p}_{3 / 2}$ 和 $\mathrm{Cu}$ $2 \mathrm{p}_{1 / 2}$ 的特征峰(图 $\left.6 \mathrm{~b}\right)^{[31]}$. 该峰的出现证明了铜的存在, 与 EDS 分析相一致. 前述红外分析表明, 分子结构中氮 元素以壳聚糖上 $\mathrm{NH}$ 与吡啶环上的 $\mathrm{N}$ 两种形式存在, 而 在图 6c 和 $6 \mathrm{~d}$ 中也给出了 PACS 螯合铜前后与红外分析 相一致的 N1s 的 XPS 能谱. 在图 6c 中, 螯合前 399 和 $398.2 \mathrm{eV}$ 分别为 $\mathrm{NH}$ 和吡啶环上 $\mathrm{N}$ 的峰 ${ }^{[32]}$. 而在螯合铜 后, 如图 6d 所示, 399 和 $398.3 \mathrm{eV}$ 处的峰为 $\mathrm{NH}$ 和吡啶 环上的 $\mathrm{N}$ 所产生，此外在 $399.6 \mathrm{eV}$ 处也有新峰产生，由 于整个螯合过程并未有 $\mathrm{N}$ 原子参与新的反应，因此推测 此峰因 $\mathrm{Cu}^{2+}$ 与 $\mathrm{N}$ 配位形成配位键所致 $(\mathrm{NCu})$.

\section{3 底物拓展}

在最佳反应条件下，将该催化体系应用到不同芳基 碘化物与吲哚衍生物和硫粉的反应中来考察催化体系 的底物适用性(表 2). 使用碘苯为底物时, 产率可以达到 88\%（3a), 而碘苯上各位置不同取代基的电子性质也会 显著影响反应的产率. 带有 $\mathrm{Me}, \mathrm{Et}, \mathrm{OMe}, \mathrm{OEt}$ 和 $\mathrm{OCF}_{3}$ 等对位供电子基团的碘苯，可以优异的收率得到相应的 产物 $(\mathbf{3 b} \sim 3 \mathbf{f})$, 而当碘苯带有 $4-\mathrm{Cl}, 4-\mathrm{NO}_{2}$ 或 $3-\mathrm{NO}_{2}$ 时, 相应产物的收率有所下降 $(\mathbf{3 h} \sim 3 \mathbf{j})$. 但是对乙酰基碘苯 

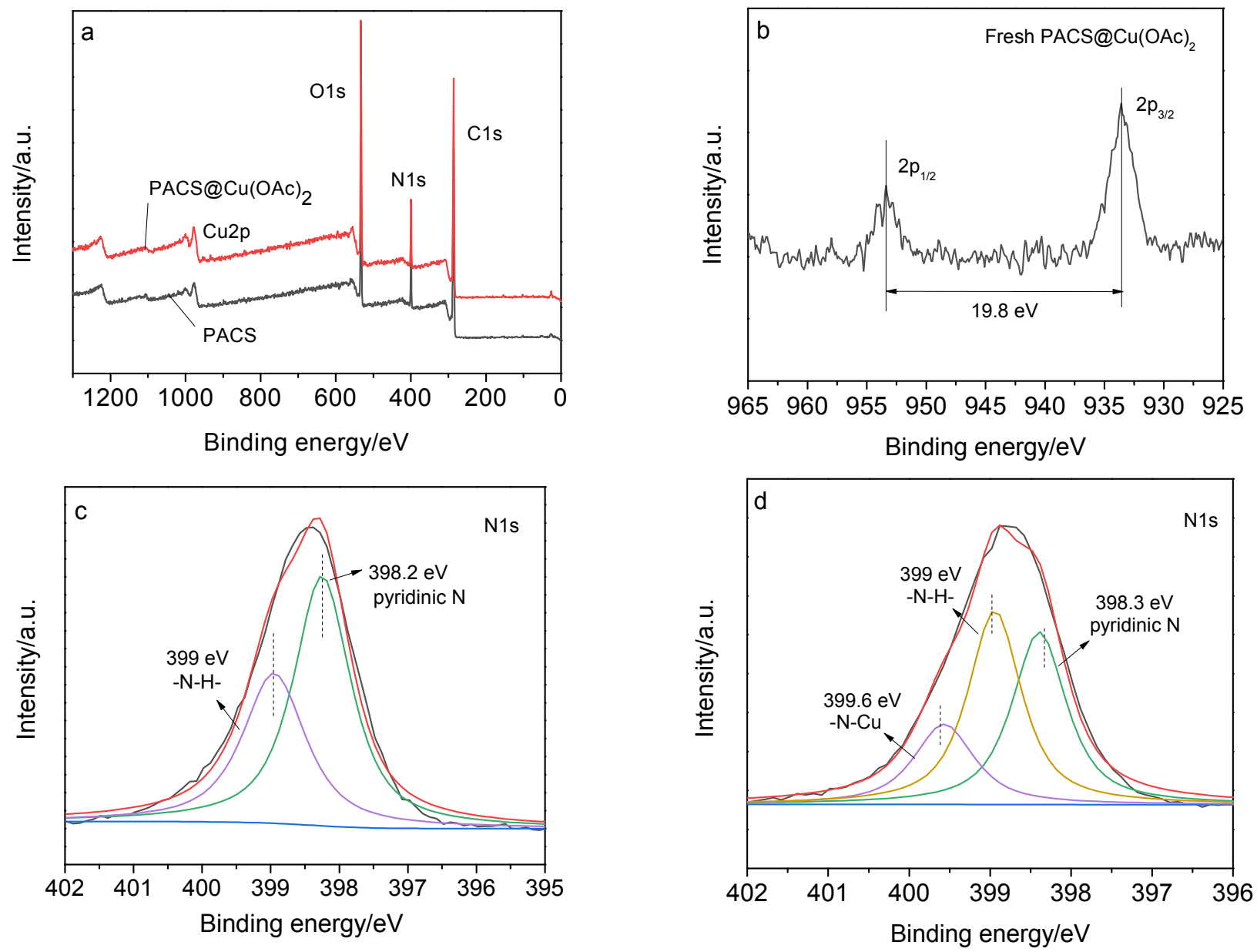

图 6 PACS 和 PACS $@ \mathrm{Cu}(\mathrm{OAc})_{2}$ 全谱(a), PACS@ $@ \mathrm{Cu}(\mathrm{OAc})_{2}$ 铜谱(b), PACS 的 N1s 谱(c)和 PACS $@ \mathrm{Cu}(\mathrm{OAc})_{2}$ 的 $\mathrm{N} 1 \mathrm{~s}$ 谱(d) Figure 6 XPS spectra of PACS and PACS@Cu(OAc) $)_{2}(a)$, copper pattern of PACS@Cu(OAc) $)_{2}(b)$, N1s pattern of PACS (c) and PACS@Cu(OAc), (d)

未能发生反应 $(\mathbf{3 k})$. 此外, 对位为氟原子的碘苯耐受性 良好，可以优异的收率得到对应产物(3g). 对于吲哚骨 架各个位置上所带有的基团, 当取代基为 $\mathrm{CN}, \mathrm{Cl}, \mathrm{NO}_{2}$ 等吸电子基时产率优异 $(\mathbf{3 I} \sim \mathbf{3 n})$, 而在 $\mathrm{C} 2, \mathrm{C} 5, \mathrm{C} 6, \mathrm{C} 7$ 位 甲基取代吲哚仅能获得 $32 \% \sim 45 \%$ 的产率 $(30 \sim 3 r)$, 这 可能是因甲基的供电子能力使芳香环电子云密度增大 而不利于碘中间体的形成所致. 最后, 对带有不同 $N$-取 代基的吲哚在反应中的适用性进行了研究, 带有苯基、 苄基和异丙基的 $N$-取代吲哚可以获得收率良好的相应 产物(3s $\sim 3 \mathbf{u})$, 而无 $N$-取代基时不能发生反应 $(\mathbf{3 v})$.

\section{4 催化剂的循环使用研究}

本工作对非均相催化剂 PACS@Cu(OAc) $)_{2}$ 的循环使 用进行了考察. 通过离心、洗涤、干燥后得到可再循环 利用的催化剂, 实验结果如图 7 所示. 当催化剂使用到 第 5 次时, 反应仍能达到 $76 \%$ 的产率, 但是相较于催化 剂首次使用, 该反应的产率略有下降. 结合前面的 EDS 表征可知, 催化效率的降低是催化剂负载的铜元素部分 流失所致.

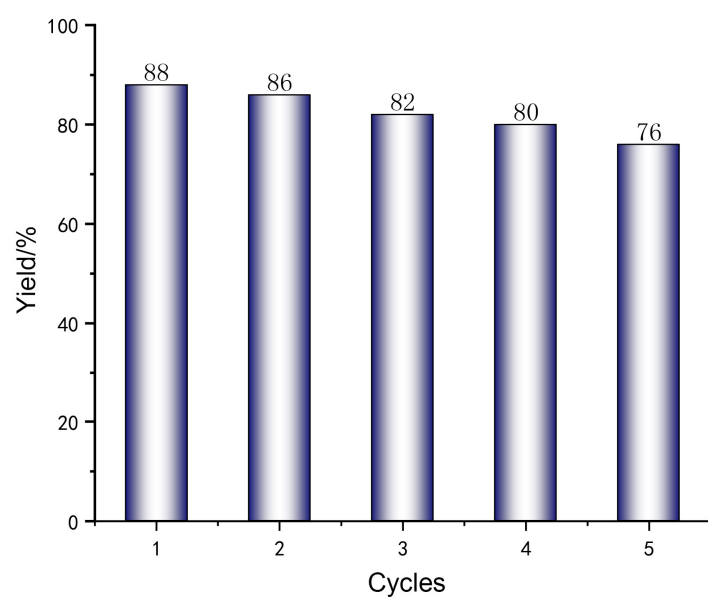

图 7 PACS@Cu(OAc) $)_{2}$ 催化剂的循环使用

Figure 7 Recycling of PACS $@ \mathrm{Cu}(\mathrm{OAc})_{2}$ catalyst

\section{2 结论}

本工作通过引入 2-吡啶甲酸对壳聚糖进行改性并 螯合醋酸铜制备了非均相催化剂 PACS@Cu(OAc) $)_{2}$, 将 其用于单质硫为硫源的吲哚 C-3 位硫醚化物的反应, 得 
表 2 PACS@ $\mathrm{Cu}(\mathrm{OAc})_{2}$ 催化吲哚 C-3 位硫醚化的反应 ${ }^{a}$

Table 2 Indole $\mathrm{C}-3$ thioetherification reaction catalyzed by PACS@ $\mathrm{Cu}(\mathrm{OAc})_{2}$

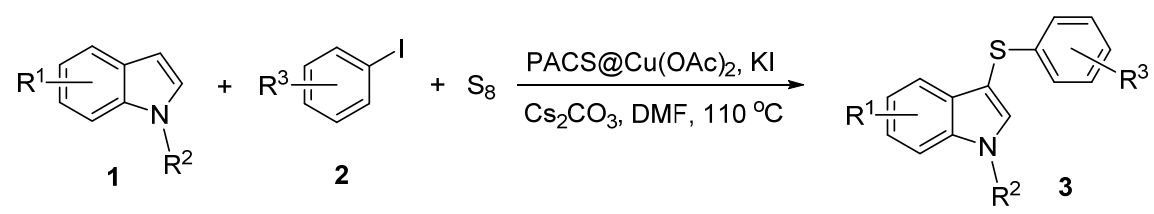

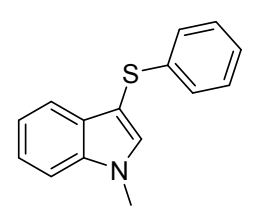

$3 a, 88 \%$

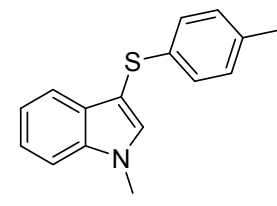

$3 b, 88 \%$<smiles>CCc1ccc(Sc2cn(C)c3ccccc23)cc1</smiles>

$3 c, 86 \%$<smiles>COc1ccc(Sc2cn(C)c3ccccc23)cc1</smiles>

$3 d, 80 \%$<smiles>CCOc1ccc(Sc2cn(C)c3ccccc23)cc1</smiles><smiles>C[13CH3]</smiles>

3j, $40 \%$<smiles>Cc1ccc(Sc2cn(C)c3ccccc23)cc1</smiles>

3k, 0\%<smiles>Cc1ccc2c(c1)c(Sc1ccccc1)cn2C</smiles>

3p, $40 \%$<smiles>Cn1cc(Sc2ccc(F)cc2)c2ccccc21</smiles>

3g, $92 \%$<smiles>Cn1cc(Sc2ccccc2)c2cc(C#N)ccc21</smiles>

3I, $85 \%$<smiles>Cc1ccc2c(Sc3ccccc3)cn(C)c2c1</smiles>

$3 q, 45 \%$<smiles>CC(C)n1cc(Sc2ccccc2)c2ccccc21</smiles>

$3 u, 86 \%$<smiles>Cn1cc(Sc2ccc(Cl)cc2)c2ccccc21</smiles>

3h, $60 \%$<smiles>Cn1cc(Sc2ccccc2)c2cc([N+](=O)[O-])ccc21</smiles>

$3 \mathrm{~m}, 83 \%$<smiles>Cc1cccc2c(Sc3ccccc3)cn(C)c12</smiles>

$3 r, 35 \%$<smiles>c1ccc(Sc2c[nH]c3ccccc23)cc1</smiles>

$3 v, 0 \%$<smiles>Cn1cc(Sc2ccc([N+](=O)[O-])cc2)c2ccccc21</smiles>

$3 i, 48 \%$<smiles>Cc1c(Sc2ccccc2)c2ccccc2n1C</smiles>

3o, $32 \%$

${ }^{a}$ Reaction conditions: 1a $(1.0 \mathrm{mmol}), 2 \mathrm{a}(2 \mathrm{mmol}), \mathrm{S}_{8}(2 \mathrm{mmol}), \mathrm{PACS} @ \mathrm{Cu}(\mathrm{OAc})_{2}(100 \mathrm{mg}), \mathrm{KI}(10 \mathrm{mmol} \%), \mathrm{Cs}_{2} \mathrm{CO}{ }_{3}(1.0 \mathrm{mmol}), \mathrm{DMF}(5 \mathrm{~mL}), 12 \mathrm{~h}, 110{ }^{\circ} \mathrm{C}$. Isolated yield.

到最优的反应条件为: $\mathrm{Cs}_{2} \mathrm{CO}_{3}$ 为碱, $\mathrm{KI}$ 为添加剂, $\mathrm{DMF}$ 为溶剂, $110{ }^{\circ} \mathrm{C}$. 该催化体系的最高收率可达 $92 \%$, 底 物适用性良好. 对 PACS@ $\mathrm{Cu}(\mathrm{OAc})_{2}$ 进行了 FT-IR、TG、 SEM、EDS、XRD、XPS 等表征与分析, 表明该催化体 系无需添加额外配体, 且催化剂易分离回收, 循环使用 5 次仍能保持较高的活性.

\section{3 实验部分}

\section{1 仪器与试剂}

${ }^{1} \mathrm{H}$ NMR $(400 \mathrm{MHz})$ 和 ${ }^{13} \mathrm{C}$ NMR (100 MHz) 采用瑞 士布鲁克公司 AVANCE III HD $400 \mathrm{MHz}$ 核磁共振仪测 定; 柱层析所用硅胶粉为 Greagent 200 300 目硅胶; 壳 聚糖粉末(mw：10000 50000，脱乙酰度 95\%)购自阿拉 丁试剂(上海)有限公司, 无需进一步纯化即可直接使用; 实验所用其他药品均为购自国药、安耐吉等公司的市售 分析纯试剂.

\section{2 实验方法}

\subsubsection{2-吡啶甲酸改性壳聚糖铜配合物的合成}

将 $N, N$-羰基二咪唑(20 mmol)和 2 -吡啶羧酸(15 $\mathrm{mmol}$ )溶于 $50 \mathrm{~mL}$ 的甲苯中 ${ }^{[33]}$, 于 $60{ }^{\circ} \mathrm{C}$ 下反应 $2 \mathrm{~h}$, 随 即加入壳聚糖粉末 $(1.61 \mathrm{~g}, 10 \mathrm{mmol})$ 并升温至 $80{ }^{\circ} \mathrm{C}$ 继 续摚拌 $12 \mathrm{~h}$. 随后待反应自然冷却到室温, 抽滤得到不 溶于甲苯的固体，倒入无水乙醇继续抽滤以洗掉附着在 
固体表面的未反应物质. 最后将清洗后的固体移至 $50{ }^{\circ} \mathrm{C}$ 真空干燥箱中放置 $12 \mathrm{~h}$, 得到粉末状 2-吡啶甲酸 改性壳聚糖(PACS).

分别取 $0.2 \mathrm{~g} \mathrm{Cu}(\mathrm{OAc})_{2}$ 和 $\mathrm{CuSO}_{4}$ 溶于 $100 \mathrm{~mL}$ 去离 子水中, 取 $0.2 \mathrm{~g} \mathrm{CuI}$ 溶于 $100 \mathrm{~mL}$ 乙腈, 加入上述 PACS 并于 $50{ }^{\circ} \mathrm{C}$ 下搅拌 $3 \mathrm{~h}$ 后, 采用离心分离收集不溶固体, 多次洗涤以去除附着在催化剂表面未参与螯合的铜盐. 随后将其移至 $50{ }^{\circ} \mathrm{C}$ 的真空干燥箱内放置 $12 \mathrm{~h}$, 得到三 种铜盐配合物 $\mathrm{PACS} @ \mathrm{Cu}(\mathrm{OAc})_{2}$ 、PACS $@ \mathrm{CuSO}_{4}$ 、 PACS@CuI, 经测定三种催化剂的铜含量分别为 0.164、 $0.208 、 0.253 \mathrm{mmol} / \mathrm{g}$.

\subsection{2 吲哚 C-3 位硫醚化反应}

向反应管内依次投入催化剂(100 mg)、 $N$-甲基吲哚 (1.0 mmol)、芳基卤化物 $(2.0 \mathrm{mmol}) 、 \mathrm{~S}_{8}(2.0 \mathrm{mmol}) 、$ 添 加剂(10 mmol $\%$ )、碱 $(1.0 \mathrm{mmol})$ 和溶剂 $(5 \mathrm{~mL})$, 在 $110{ }^{\circ} \mathrm{C}$ 下搅拌 $12 \mathrm{~h}$ 后冷却至室温. 将反应液移入分液漏斗, 并 使用 $15 \mathrm{~mL}$ 乙酸乙酯和 $15 \mathrm{~mL}$ 水进行萃取, 收集上层乙 酸乙酯相, 加入无水硫酸钠进一步除去残余的水, 抽滤, 将所得滤液使用旋转蒸发仪除去乙酸乙酯, 初步获得粗 产品. 以石油醚和乙酸乙酯的混合溶液为流动相, 通过 柱层析提纯进一步获得相应的纯品，计算收率.

1-甲基-3-(苯硫基)- $1 H$-吲哚(3a)：白色固体，产率 $88 \%$. m.p. $84 \sim 86{ }^{\circ} \mathrm{C}$ (lit. $\left.{ }^{[34]} 85 \sim 87{ }^{\circ} \mathrm{C}\right) ;{ }^{1} \mathrm{H}$ NMR $(400$ $\left.\mathrm{MHz} \mathrm{CDCl}_{3}\right) \delta: 7.64(\mathrm{~d}, J=8.0 \mathrm{~Hz}, 1 \mathrm{H}), 7.42(\mathrm{~d}, J=8.2$ $\mathrm{Hz}, 1 \mathrm{H}), 7.37(\mathrm{~s}, 1 \mathrm{H}), 7.36 \sim 7.29(\mathrm{~m}, 1 \mathrm{H}), 7.23 \sim 7.15(\mathrm{~m}$, $3 \mathrm{H}), 7.15 \sim 7.10(\mathrm{~m}, 2 \mathrm{H}), 7.10 \sim 7.03(\mathrm{~m}, 1 \mathrm{H}), 3.88(\mathrm{~s}$, $3 \mathrm{H}) ;{ }^{13} \mathrm{C} \mathrm{NMR}\left(100 \mathrm{MHz}, \mathrm{CDCl}_{3}\right) \delta: 139.7,137.6,135.0$, $129.9,128.6,125.8,124.7,122.6,120.5,119.8,109.7$, 100.6, 30.9. HRMS (ESI) calcd for $\mathrm{C}_{15} \mathrm{H}_{14} \mathrm{NS}[\mathrm{M}+\mathrm{H}]^{+}$ 240.0847; found 240.0870.

1-甲基-3-(4-甲基苯硫基)- $1 H$-吲哚(3b) ${ }^{[34]}$ : 白色固 体, 产率 $88 \%$. m.p. $122 \sim 123{ }^{\circ} \mathrm{C}$ (lit. ${ }^{[34]} 122 \sim 123{ }^{\circ} \mathrm{C}$ ); ${ }^{1} \mathrm{H}$ NMR $\left(400 \mathrm{MHz}, \mathrm{CDCl}_{3}\right) \delta: 7.67 \sim 7.65(\mathrm{~m}, 1 \mathrm{H}), 7.41$ $(\mathrm{d}, J=8.2 \mathrm{~Hz}, 1 \mathrm{H}), 7.36 \sim 7.30(\mathrm{~m}, 2 \mathrm{H}), 7.22 \sim 7.28(\mathrm{~m}$, $1 \mathrm{H}), 7.10 \sim 7.04(\mathrm{~m}, 2 \mathrm{H}), 7.01(\mathrm{~d}, J=8.0 \mathrm{~Hz}, 2 \mathrm{H}), 3.86(\mathrm{~s}$, $3 \mathrm{H}), 2.29(\mathrm{~s}, 3 \mathrm{H}) ;{ }^{13} \mathrm{C}$ NMR $\left(100 \mathrm{MHz}, \mathrm{CDCl}_{3}\right) \delta: 136.5$, $134.9,133.8,133.5,128.8,128.4,125.1,121.4,119.4$, 118.7, 108.6, 100.1, 32.1, 19.8. HRMS (ESI) calcd for $\mathrm{C}_{16} \mathrm{H}_{15} \mathrm{NS}[\mathrm{M}+\mathrm{H}]^{+}$253.0925; found 253.0947.

3-[(4-乙基苯基)硫基]-1-甲基- $1 H$-吲哚(3c): 白色固 体, 产率 86\%. m.p. 133 135 ${ }^{\circ} \mathrm{C} ;{ }^{1} \mathrm{H}$ NMR (400 MHz, $\left.\mathrm{CDCl}_{3}\right) \delta: 7.72 \sim 7.64(\mathrm{~m}, 1 \mathrm{H}), 7.50(\mathrm{~d}, J=8.4 \mathrm{~Hz}, 1 \mathrm{H})$, $7.41(\mathrm{~d}, J=8.2 \mathrm{~Hz}, 1 \mathrm{H}), 7.23 \sim 7.18(\mathrm{~m}, 2 \mathrm{H}), 7.11(\mathrm{~d}, J=$ $8.4 \mathrm{~Hz}, 2 \mathrm{H}), 7.03$ (d, $J=8.4 \mathrm{~Hz}, 2 \mathrm{H}), 3.87$ (s, 3H), 2.58 (q, $J=7.6 \mathrm{~Hz}, 2 \mathrm{H}), 1.20(\mathrm{t}, J=7.6 \mathrm{~Hz}, 3 \mathrm{H}) ;{ }^{13} \mathrm{C} \mathrm{NMR}(100$
$\left.\mathrm{MHz}, \mathrm{CDCl}_{3}\right) \delta: 141.0,134.9,130.9,129.9,128.5,128.3$, $126.2,122.5,120.4,19.8,109.7,101.3,33.1,28.3,15.6$. HRMS (ESI) calcd for $\mathrm{C}_{17} \mathrm{H}_{18} \mathrm{~S}[\mathrm{M}+\mathrm{H}]^{+}$268.1160; found 268.1179 .

3-[(4-甲氧基苯基)硫基]-1-甲基- $1 H$-吲哚(3d): 白色 固体，产率 80\%. m.p. $60 \sim 61{ }^{\circ} \mathrm{C}$ (lit. ${ }^{[34]} 60 \sim 62{ }^{\circ} \mathrm{C}$ ); ${ }^{1} \mathrm{H}$ NMR (400 MHz, $\left.\mathrm{CDCl}_{3}\right) \delta: 7.73(\mathrm{~d}, J=8.0 \mathrm{~Hz}, 1 \mathrm{H}), 7.42$ $(\mathrm{d}, J=8.2 \mathrm{~Hz}, 1 \mathrm{H}), 7.39 \sim 7.31(\mathrm{~m}, 2 \mathrm{H}), 7.29 \sim 7.16(\mathrm{~m}$, $3 \mathrm{H}), 6.83 \sim 6.77(\mathrm{~m}, 2 \mathrm{H}), 3.83(\mathrm{~s}, 3 \mathrm{H}), 3.78(\mathrm{~s}, 3 \mathrm{H}) ;{ }^{13} \mathrm{C}$ NMR (100 MHz, $\left.\mathrm{CDCl}_{3}\right) \delta: 157.8,137.6,134.6,130.1$, $129.8,128.5,122.6,120.5,119.8,114.6,109.8,102.4$, 55.4, 33.1. HRMS (ESI) calcd for $\mathrm{C}_{16} \mathrm{H}_{16} \mathrm{OS}[\mathrm{M}+\mathrm{H}]^{+}$ 269.0874; found 269.0881.

3-[(4-乙氧基苯基)硫基]-1-甲基- $1 H$-吲哚(3e): 白色 固体, 产率 83\%. m.p. 70 72 ${ }^{\circ} \mathrm{C} ;{ }^{1} \mathrm{H}$ NMR (400 MHz, DMSO-d $\left.d_{6}\right) \delta: 7.73(\mathrm{~s}, 1 \mathrm{H}), 7.53(\mathrm{~d}, J=8.0 \mathrm{~Hz}, 1 \mathrm{H}), 7.44$ $(\mathrm{d}, J=8.0 \mathrm{~Hz}, 1 \mathrm{H}), 7.28 \sim 7.19(\mathrm{~m}, 1 \mathrm{H}), 7.16 \sim 7.03(\mathrm{~m}$, $3 \mathrm{H}), 6.86 \sim 6.72(\mathrm{~m}, 2 \mathrm{H}), 3.92(\mathrm{q}, J=7.0 \mathrm{~Hz}, 2 \mathrm{H}), 3.84(\mathrm{~s}$, $3 \mathrm{H}), 1.27(\mathrm{t}, J=7.0 \mathrm{~Hz}, 3 \mathrm{H}) ;{ }^{13} \mathrm{C} \mathrm{NMR}(100 \mathrm{MHz}$, DMSO- $\left.d_{6}\right) \delta: 157.2,137.7,136.0,129.5,128.8,122.6$, 120.6, 119.0, 115.6, 111.0, 100.9, 63.6, 33.2, 15.1. HRMS (ESI) calcd for $\mathrm{C}_{17} \mathrm{H}_{17} \mathrm{NOS}[\mathrm{M}+\mathrm{H}]^{+}$283.1031; found 283.1054 .

1-甲基-3-\{[4-(三氟甲氧基)苯基]硫基 $\}-1 H$-吲哚(3f): 白色固体，产率 $83 \%$. ${ }^{1} \mathrm{H}$ NMR (400 MHz, DMSO- $\left.d_{6}\right) \delta$ : $7.81(\mathrm{~s}, 1 \mathrm{H}), 7.58(\mathrm{~d}, J=8.0 \mathrm{~Hz}, 1 \mathrm{H}), 7.42(\mathrm{~d}, J=8.0 \mathrm{~Hz}$, $1 \mathrm{H}), 7.32 \sim 7.25(\mathrm{~m}, 1 \mathrm{H}), 7.22(\mathrm{~d}, J=8.2 \mathrm{~Hz}, 2 \mathrm{H}), 7.18 \sim$ $7.05(\mathrm{~m}, 3 \mathrm{H}), 3.88(\mathrm{~s}, 3 \mathrm{H}) ;{ }^{13} \mathrm{C}$ NMR $(100 \mathrm{MHz}$, DMSO- $\left.d_{6}\right) \delta: 146.2,139.3,137.8,136.9,129.3,127.2$, $122.8,122.2,121.0,120.5$ (q, $J=254 \mathrm{~Hz}$ ), 118.8, 111.3, 98.1, 33.3. HRMS (ESI) calcd for $\mathrm{C}_{16} \mathrm{H}_{13} \mathrm{~F}_{3} \mathrm{NOS}[\mathrm{M}+\mathrm{H}]^{+}$ 324.0670; found 324.0682.

3-[(4-氟苯基)硫基]-1-甲基- $1 H$-吲哚(3g)：白色固体, 产率 92\%. m.p. 55 58 ${ }^{\circ} \mathrm{C}$ (lit. ${ }^{[34]} 56 \sim 58{ }^{\circ} \mathrm{C}$ ); ${ }^{1} \mathrm{H}$ NMR $\left(600 \mathrm{MHz}, \mathrm{CDCl}_{3}\right) \delta: 7.62(\mathrm{~d}, J=8.0 \mathrm{~Hz}, 1 \mathrm{H}), 7.41(\mathrm{~d}, J=$ $8.0 \mathrm{~Hz}, 1 \mathrm{H}), 7.36(\mathrm{~s}, 1 \mathrm{H}), 7.35 \sim 7.29(\mathrm{~m}, 1 \mathrm{H}), 7.23 \sim 7.17$ $(\mathrm{m}, 1 \mathrm{H}), 7.15 \sim 7.07(\mathrm{~m}, 2 \mathrm{H}), 6.92 \sim 6.85(\mathrm{~m}, 2 \mathrm{H}), 3.87(\mathrm{~s}$, $3 \mathrm{H}) ;{ }^{13} \mathrm{C}$ NMR $\left(150 \mathrm{MHz}, \mathrm{CDCl}_{3}\right) \delta: 160.8$ (d, $J=246$ $\mathrm{Hz}$ ), 139.2, 136.2, 131.6, 128.8, 125.7 (d, J=19.5 Hz), $124.9,122.3,119.4,114.3,111.3,100.3,33.4$. HRMS (ESI) calcd for $\mathrm{C}_{15} \mathrm{H}_{13} \mathrm{FNS}[\mathrm{M}+\mathrm{H}]^{+}$258.0753; found 258.0768

3-[(4-氯苯基)硫基]-1-甲基- $1 H$-吲哚(3h): 白色固体, 产率 60\%. m.p. $136 \sim 139{ }^{\circ} \mathrm{C}$ (lit. ${ }^{[34]} 137 \sim 138{ }^{\circ} \mathrm{C}$ ); ${ }^{1} \mathrm{H}$ NMR (400 MHz, $\left.\mathrm{CDCl}_{3}\right) \delta: 7.60(\mathrm{~d}, J=8.0 \mathrm{~Hz}, 1 \mathrm{H}), 7.42$ 
(d, $J=8.0 \mathrm{~Hz}, 1 \mathrm{H}), 7.39 \sim 7.31(\mathrm{~m}, 2 \mathrm{H}), 7.25 \sim 7.17(\mathrm{~m}$, $1 \mathrm{H}), 7.17 \sim 7.11(\mathrm{~m}, 2 \mathrm{H}), 7.09 \sim 6.99(\mathrm{~m}, 2 \mathrm{H}), 3.88(\mathrm{~s}$, $3 \mathrm{H}) ;{ }^{13} \mathrm{C}$ NMR $\left(100 \mathrm{MHz}, \mathrm{CDCl}_{3}\right) \delta: 138.3,137.6,135.1$, $130.5,129.6,128.7,127.0,122.7,120.7,119.6,109.8$, 100.2, 33.16 HRMS (ESI) calcd for $\mathrm{C}_{15} \mathrm{H}_{13} \mathrm{ClNS}[\mathrm{M}+\mathrm{H}]^{+}$ 274.0457; found 274.0461.

1-甲基-3-[(4-硝基苯基)硫基]- $1 H$-吲哚(3i $)^{[35]}$ : 黄色 油状物, 产率 $48 \% .{ }^{1} \mathrm{H}$ NMR $\left(400 \mathrm{MHz}, \mathrm{CDCl}_{3}\right) \delta: 8.06 \sim$ $7.96(\mathrm{~m}, 2 \mathrm{H}), 7.55(\mathrm{~d}, J=8.0 \mathrm{~Hz}, 1 \mathrm{H}), 7.47(\mathrm{~d}, J=8.0 \mathrm{~Hz}$, $1 \mathrm{H}), 7.43 \sim 7.34(\mathrm{~m}, 2 \mathrm{H}), 7.26 \sim 7.20(\mathrm{~m}, 1 \mathrm{H}), 7.18 \sim 7.10$ (m, 2H), $3.92(\mathrm{~s}, 3 \mathrm{H}) ;{ }^{13} \mathrm{C}$ NMR $\left(100 \mathrm{MHz}, \mathrm{CDCl}_{3}\right) \delta$ : $150.2,144.9,137.7,135.4,129.2,125.0,123.8,123.1$, 121.1, 119.3, 110.1, 97.9, 33.3. HRMS (ESI) calcd for $\mathrm{C}_{15} \mathrm{H}_{13} \mathrm{~N}_{2} \mathrm{O}_{2} \mathrm{~S}[\mathrm{M}+\mathrm{H}]^{+}$285.0698; found 285.0711.

1-甲基-3-[(3-硝基苯基)硫基]- $1 H$-吲哚(3j): 黄色液 体, 产率 40\%. ${ }^{1} \mathrm{H}$ NMR $\left(600 \mathrm{MHz}, \mathrm{CDCl}_{3}\right) \delta: 7.94$ (t, $J=$ $2.0 \mathrm{~Hz}, 1 \mathrm{H}), 7.91 \sim 7.89(\mathrm{~m}, 1 \mathrm{H}), 7.57(\mathrm{~d}, J=8.0 \mathrm{~Hz}, 1 \mathrm{H})$, $7.45(\mathrm{~d}, J=8.2 \mathrm{~Hz}, 1 \mathrm{H}), 7.42(\mathrm{~s}, 1 \mathrm{H}), 7.40 \sim 7.36(\mathrm{~m}, 1 \mathrm{H})$, $7.36 \sim 7.30(\mathrm{~m}, 2 \mathrm{H}), 7.21(\mathrm{t}, J=7.4 \mathrm{~Hz}, 1 \mathrm{H}), 3.92(\mathrm{~s}, 3 \mathrm{H})$; ${ }^{13} \mathrm{C}$ NMR $\left(150 \mathrm{MHz}, \mathrm{CDCl}_{3}\right) \delta: 148.7,143.0,137.7,35.5$, $131.2,129.3,123.0,121.0,120.2,119.6,119.3,110.1$, 98.5, 33.4. HRMS (ESI) calcd for $\mathrm{C}_{15} \mathrm{H}_{13} \mathrm{~N}_{2} \mathrm{O}_{2} \mathrm{~S}[\mathrm{M}+\mathrm{H}]^{+}$ 285.0698; found 285.0705.

1-甲基-5-氧基-3-(苯硫基)- $1 H$-吲哚(3I)：白色固体, 产率 85\%. m.p. $155 \sim 158{ }^{\circ} \mathrm{C} ;{ }^{1} \mathrm{H}$ NMR $(400 \mathrm{MHz}$, $\left.\mathrm{CDCl}_{3}\right) \delta: 7.96(\mathrm{~s}, 1 \mathrm{H}), 7.55 \sim 7.50(\mathrm{~m}, 1 \mathrm{H}), 7.49 \sim 7.43$ (m, 2H), $7.25 \sim 7.19(\mathrm{~m}, 2 \mathrm{H}), 7.15 \sim 7.08(\mathrm{~m}, 3 \mathrm{H}), 3.91(\mathrm{~s}$, $3 \mathrm{H}) ;{ }^{13} \mathrm{C}$ NMR (100 MHz, $\left.\mathrm{CDCl}_{3}\right) \delta: 139.1,138.2,137.0$, $129.6,128.9,126.3,125.6,125.4,125.3,120.3,110.8$, 103.8, 103.1, 33.4. HRMS (ESI) calcd for $\mathrm{C}_{16} \mathrm{H}_{13} \mathrm{~N}_{2} \mathrm{~S}[\mathrm{M}+$ $\mathrm{H}]^{+}$265.0799; found 265.0810.

1-甲基-5-硝基-3-(苯硫基)-1 $H$-吲哚(3m): 黄色固体, 产率 83\%. m.p. $184 \sim 185{ }^{\circ} \mathrm{C}$ (lit. ${ }^{[17]} 183 \sim 185{ }^{\circ} \mathrm{C}$ ); ${ }^{1} \mathrm{H}$ NMR (400 MHz, $\left.\mathrm{CDCl}_{3}\right) \delta: 8.57(\mathrm{~d}, J=2.2 \mathrm{~Hz}, 1 \mathrm{H}), 8.20$ (dd, $J=9.2,2.0 \mathrm{~Hz}, 1 \mathrm{H}), 7.51$ (s, 1H), $7.43(\mathrm{~d}, J=9.2 \mathrm{~Hz}$, $1 \mathrm{H}), 7.24 \sim 7.17(\mathrm{~m}, 2 \mathrm{H}), 7.16 \sim 7.09(\mathrm{~m}, 3 \mathrm{H}), 3.93(\mathrm{~s}$, $3 \mathrm{H}) ;{ }^{13} \mathrm{C}$ NMR (100 MHz, $\left.\mathrm{CDCl}_{3}\right) \delta: 141.5,139.2,137.1$, $137.1,128.3,127.9,125.3,124.4,117.2,115.9,109.0$, 103.7, 32.6. HRMS (ESI) calcd for $\mathrm{C}_{15} \mathrm{H}_{13} \mathrm{~N}_{2} \mathrm{O}_{2} \mathrm{~S}[\mathrm{M}+\mathrm{H}]^{+}$ 285.0698; found 285.0705.

1-甲基-5-氯-3-(苯硫基)- $1 H$-吲哚(3n): 白色固体, 产率 84\%. m.p. $136 \sim 138{ }^{\circ} \mathrm{C}$ (lit. ${ }^{[36]} 136 \sim 138{ }^{\circ} \mathrm{C}$ ); ${ }^{1} \mathrm{H}$ NMR (400 MHz, $\left.\mathrm{CDCl}_{3}\right) \delta: 7.63(\mathrm{~d}, J=2.0 \mathrm{~Hz}, 1 \mathrm{H}), 7.35$ (s, 1H), $7.30(\mathrm{~d}, \quad J=7.6 \mathrm{~Hz}, 1 \mathrm{H}), 7.27(\mathrm{~d}, J=2.0 \mathrm{~Hz}, 1 \mathrm{H})$, $7.20(\mathrm{~m}, 2 \mathrm{H}), 7.12(\mathrm{~m}, 3 \mathrm{H}), 3.83(\mathrm{~s}, 3 \mathrm{H}) ;{ }^{13} \mathrm{C}$ NMR $(100$
$\left.\mathrm{MHz}, \mathrm{CDCl}_{3}\right) \delta: 139.2,136.4,136.0,131.0,128.8,126.7$, $125.8,125.0,123.0,119.2,111.0,100.5,33.4$. HRMS (ESI) calcd for $\mathrm{C}_{15} \mathrm{H}_{13} \mathrm{ClNS}[\mathrm{M}+\mathrm{H}]^{+}$274.0457; found 274.0469 .

1,2-二甲基-3-(苯硫基)- $1 H$-吲哚(3o)：白色固体，产 率 32\%. m.p. $109 \sim 110{ }^{\circ} \mathrm{C} \quad\left(\right.$ lit. ${ }^{[36]} 108 \sim 110{ }^{\circ} \mathrm{C}$ ); ${ }^{1} \mathrm{H}$ NMR $\left(400 \mathrm{MHz}, \mathrm{CDCl}_{3}\right) \delta: 7.69 \sim 7.55(\mathrm{~m}, 1 \mathrm{H}), 7.38(\mathrm{~d}$, $J=8.0 \mathrm{~Hz}, 1 \mathrm{H}), 7.33 \sim 7.24(\mathrm{~m}, 1 \mathrm{H}), 7.23 \sim 7.14(\mathrm{~m}, 3 \mathrm{H})$, $7.14 \sim 7.02(\mathrm{~m}, 3 \mathrm{H}), 3.80(\mathrm{~s}, 3 \mathrm{H}), 2.56(\mathrm{~s}, 3 \mathrm{H}) ;{ }^{13} \mathrm{C} \mathrm{NMR}$ $\left(150 \mathrm{MHz}, \mathrm{CDCl}_{3}\right) \delta: 142.9,139.8,137.1,129.8,128.6$, 125.4, 124.4, 121.8, 120.5, 119.0, 109.0, 98.0, 30.34, 10.9. HRMS (ESI) calcd for $\mathrm{C}_{16} \mathrm{H}_{16} \mathrm{NS}[\mathrm{M}+\mathrm{H}]^{+}$254.1003; found 254.1021 .

1,5-二甲基-3-(苯硫基)- $1 H$-吲哚(3p): 黄色油状物, 产率 40\%. ${ }^{1} \mathrm{H}$ NMR $\left(400 \mathrm{MHz}, \mathrm{CDCl}_{3}\right) \delta: 7.52(\mathrm{~d}, J=8.1$ $\mathrm{Hz}, 1 \mathrm{H}), 7.29$ (d, $J=3.5 \mathrm{~Hz}, 1 \mathrm{H}), 7.22$ (s, 1H), 7.16 (ddd, $J=10.1,8.5,6.4 \mathrm{~Hz}, 4 \mathrm{H}), 7.10 \sim 7.01(\mathrm{~m}, 2 \mathrm{H}), 3.84(\mathrm{~s}$, $3 \mathrm{H}), 2.55(\mathrm{~s}, 3 \mathrm{H}) ;{ }^{13} \mathrm{C}$ NMR $\left(100 \mathrm{MHz}, \mathrm{CDCl}_{3}\right) \delta: 139.9$, $138.0,134.5,132.6,129.2,128.6,125.7,124.6,122.3$, 119.4, 109.7, 100.4, 33.0, 21.9. HRMS (ESI) calcd for $\mathrm{C}_{16} \mathrm{H}_{16} \mathrm{NS}[\mathrm{M}+\mathrm{H}]^{+}$254.1003; found 254.1021.

1,6-二甲基-3-(苯硫基)-1 $H$-吲哚(3q)：黄色油状物， 产率 45\%. ${ }^{1} \mathrm{H}$ NMR $\left(600 \mathrm{MHz}, \mathrm{CDCl}_{3}\right) \delta: 7.50(\mathrm{~d}, J=8.4$ $\mathrm{Hz}, 1 \mathrm{H}), 7.29$ (s, 1H), $7.21(\mathrm{~s}, 1 \mathrm{H}), 7.19 \sim 7.14(\mathrm{~m}, 2 \mathrm{H})$, $7.13 \sim 7.10(\mathrm{~m}, 2 \mathrm{H}), 7.08 \sim 7.04(\mathrm{~m}, 1 \mathrm{H}), 7.04 \sim 7.01(\mathrm{~m}$, $1 \mathrm{H}), 3.84(\mathrm{~s}, 3 \mathrm{H}), 2.54(\mathrm{~s}, 3 \mathrm{H}) ;{ }^{13} \mathrm{C}$ NMR $(150 \mathrm{MHz}$, $\left.\mathrm{CDCl}_{3}\right) \delta: 139.8,138.0,134.5,132.5,128.6,127.7,125.6$, 124.6, 122.3, 119.4, 109.7, 100.0, 33.1, 21.9. HRMS (ESI) calcd for $\mathrm{C}_{16} \mathrm{H}_{16} \mathrm{NS}[\mathrm{M}+\mathrm{H}]^{+}$254.1003; found 254.1021.

1,7-二甲基-3-(苯硫基)- $1 H$-吲哚(3r): 黄色油状物, 产率 35\%. ${ }^{1} \mathrm{H}$ NMR $\left(400 \mathrm{MHz}, \mathrm{CDCl}_{3}\right) \delta: 7.48(\mathrm{~d}, J=7.6$ $\mathrm{Hz}, 1 \mathrm{H}), 7.25(\mathrm{~s}, 1 \mathrm{H}), 7.21 \sim 7.15(\mathrm{~m}, 2 \mathrm{H}), 7.15 \sim 7.10(\mathrm{~m}$, $2 \mathrm{H}), 7.10 \sim 6.98(\mathrm{~m}, 3 \mathrm{H}), 4.13(\mathrm{~s}, 3 \mathrm{H}), 2.83(\mathrm{~s}, 3 \mathrm{H}) ;{ }^{13} \mathrm{C}$ NMR $\left(100 \mathrm{MHz}, \mathrm{CDCl}_{3}\right) \delta: 138.6,135.6,135.2,129.9$, 127.6, 124.6, 124.2, 123.6, 120.7, 119.7, 116.9, 99.1, 36.1, 18.6. HRMS (ESI) calcd for $\mathrm{C}_{16} \mathrm{H}_{16} \mathrm{NS}[\mathrm{M}+\mathrm{H}]$ 254.1003; found 254.1021.

1-苯基-3-(苯硫基)- $1 H$-吲哚(3s) ${ }^{[17]}$ : 黄色油状物, 产 率 55\%. ${ }^{1} \mathrm{H}$ NMR $\left(400 \mathrm{MHz}, \mathrm{CDCl}_{3}\right) \delta: 7.75 \sim 7.62(\mathrm{~m}$, $3 \mathrm{H}), 7.62 \sim 7.56(\mathrm{~m}, 4 \mathrm{H}), 7.49 \sim 7.42(\mathrm{~m}, 1 \mathrm{H}), 7.33(\mathrm{dd}$, $J=8.0,7.2 \mathrm{~Hz}, 1 \mathrm{H}), 7.30 \sim 7.19(\mathrm{~m}, 5 \mathrm{H}), 7.17 \sim 7.09(\mathrm{~m}$, $1 \mathrm{H}) ;{ }^{13} \mathrm{C}$ NMR $\left(100 \mathrm{MHz}, \mathrm{CDCl}_{3}\right) \delta: 137.9,137.8,135.8$, $132.8,129.2,128.8,127.7,126.1,125.1,123.9,123.4$, 122.3, 120.3, 119.0, 110.0, 102.9. HRMS (ESI) calcd for $\mathrm{C}_{20} \mathrm{H}_{16} \mathrm{NS}[\mathrm{M}+\mathrm{H}]^{+}$302.1003; found 302.1018. 
1-苠基-3-(苯硫基)- $1 H$-吲哚(3t) ${ }^{[37]}$ : 无色油状物, 产 率 76\%. ${ }^{1} \mathrm{H}$ NMR $\left(600 \mathrm{MHz}, \mathrm{CDCl}_{3}\right) \delta: 7.66(\mathrm{~d}, J=7.8$ $\mathrm{Hz}, 1 \mathrm{H}), 7.44(\mathrm{~s}, 1 \mathrm{H}), 7.40 \sim 7.35(\mathrm{~m}, 3 \mathrm{H}), 7.35 \sim 7.31(\mathrm{~m}$, $1 \mathrm{H}), 7.30 \sim 7.27(\mathrm{~m}, 1 \mathrm{H}), 7.20 \sim 7.19(\mathrm{~m}, 4 \mathrm{H}), 7.18(\mathrm{~s}$, $1 \mathrm{H}), 7.15(\mathrm{~d}, J=1.2 \mathrm{~Hz}, 1 \mathrm{H}), 7.14(\mathrm{~d}, J=1.2 \mathrm{~Hz}, 1 \mathrm{H})$, $7.10 \sim 7.06(\mathrm{~m}, 1 \mathrm{H}), 5.39(\mathrm{~s}, 2 \mathrm{H}) ;{ }^{13} \mathrm{C}$ NMR $(150 \mathrm{MHz}$, $\left.\mathrm{CDCl}_{3}\right) \delta: 139.5,137.2,136.6,134.5,130.1,129.0,128.7$, $128.0,127.0,125.8,124.7,122.8,120.8,119.9,110.3$, 101.5, 50.5. HRMS (ESI) calcd for $\mathrm{C}_{21} \mathrm{H}_{18} \mathrm{NS}[\mathrm{M}+\mathrm{H}]^{+}$ 316.1160 ; found 316.1174 .

1-异丙基-3-(苯硫基)-1 $H$-吲哚(3u)：黄色油状物，产 率 86\%. ${ }^{1} \mathrm{H}$ NMR (400 MHz, $\left.\mathrm{CDCl}_{3}\right) \delta: 7.68(\mathrm{~d}, J=8.0$ $\mathrm{Hz}, 1 \mathrm{H}), 7.56(\mathrm{~s}, 1 \mathrm{H}), 7.50(\mathrm{~d}, J=8.4 \mathrm{~Hz}, 1 \mathrm{H}), 7.33(\mathrm{t}, J=$ $7.6 \mathrm{~Hz}, 1 \mathrm{H}), 7.21 \sim 7.19(\mathrm{~m}, 3 \mathrm{H}), 7.17 \sim 7.12(\mathrm{~m}, 2 \mathrm{H})$, $7.11 \sim 7.07(\mathrm{~m}, 1 \mathrm{H}), 4.77(\mathrm{~m}, J=6.8 \mathrm{~Hz}, 1 \mathrm{H}), 1.62(\mathrm{~d}, J=$ $6.8 \mathrm{~Hz}, 6 \mathrm{H}) ;{ }^{13} \mathrm{C}$ NMR $\left(100 \mathrm{MHz}, \mathrm{CDCl}_{3}\right) \delta: 140.0,136.6$, $130.4,130.1,128.8,125.8,124.7,122.5,120.6,120.0$, 110.2, 100.8, 47.8. HRMS (ESI) calcd for $\mathrm{C}_{17} \mathrm{H}_{18} \mathrm{NS}[\mathrm{M}+$ $\mathrm{H}]^{+}$268.1160; found 268.1176.

辅助材料(Supporting Information) 所合成目标化合 物的 ${ }^{1} \mathrm{H}$ NMR 和 ${ }^{13} \mathrm{C}$ NMR 谱. 这些材料可以免费从本刊 网站(http://sioc-journal.cn/)上下载.

\section{References}

[1] (a) Chung, W. J.; Griebel, J. J.; Kim, E. T.; Yoon, H.; Simmonds, A. G.; Ji, H. J.; Dirlam, P. T.; Glass, R. S.; Wie, J. J.; Nguyen, N. A.; Guralnick, B. W.; Park, J.; Somogyi, A.; Theato, P.; Mackay, M. E.; Sung, Y.-E.; Char, K.; Pyun, J. Nat. Chem. 2013, 5, 518.

(b) Horita, Y.; Takii, T.; Chiba, T.; Kuroishi, R.; Maeda, Y.; Kurono, Y.; Onozaki, K. Bioorg. Med. Chem. Lett. 2009, 19, 6313.

(c) Ruan, L.; Fan, R.; Liu, X.; Chen, J.; Weng, J. Chin. J. Org. Chem. 2015, 35, 1166 (in Chinese).

(阮铃莉, 范人杰, 刘幸海, 陈杰, 翁建全, 有机化学, 2015, 35, 1166.)

(d) Lee, E.-J.; Kim, K.-W.; Kim, G.-H.; Park, C.-E.; Choi, J.-H. Fibers Polym. 2018, 19, 1791.

[2] (a) Dunbar, K. L.; Scharf, D. H.; Litomska, A.; Hertweck, C. Chem. Rev. 2017, 117, 5521.

(b) Chekler, E. L. P.; Elokdah, H. M.; Butera, J. Tetrahedron Lett. 2008, 49, 6709 .

[3] (a) Pourshab, M.; Asghari, S.; Mohseni, M. J. Heterocycl. Chem. 2018, 55, 173 .

(b) Zhang, B.; Kang, Y.; Shi, R. Chin. J. Org. Chem. 2016, 36, 1814 (in Chinese). (张变香, 穴永强, 史瑞雪, 有机化学, 2016, 36, 1814.)

[4] Funk, C. D. Nat. Rev. Drug Discovery 2005, 4, 664.

[5] La Regina, G.; Edler, M. C.; Brancale, A.; Kandil, S.; Coluccia, A.; Piscitelli, F.; Hamel, E.; De Martino, G.; Matesanz, R.; Diaz, J. F.; Scovassi, A. I.; Prosperi, E.; Lavecchia, A.; Novellino, E.; Artico, M.; Silvestri, R. J. Med. Chem. 2007, 50, 2865.

[6] La Regina, G.; Coluccia, A.; Brancale, A.; Piscitelli, F.; Famiglini, V.; Cosconati, S.; Maga, G.; Samuele, A.; Gonzalez, E.; Clotet, B.; Schols, D.; Este, J. A.; Novellino, E.; Silvestri, R. J. Med. Chem. 2012, 55,6634

[7] Lavekar, A. G.; Equbal, D.; Saima; Sinha, A. K. Adv. Synth. Catal.
2018, 360, 180.

[8] Gandeepan, P.; Koeller, J.; Ackermann, L. ACS Catal. 2017, 7, 1030.

[9] Liu, X. X.; Cui, H. H.; Yang, D. S.; Dai, S. C.; Zhang, G. Q.; Wei, W.; Wang, H. Catal. Lett. 2016, 146, 1743.

[10] Luckhurst, C. A.; Millichip, I.; Parker, B.; Reuberson, J.; Furber, M. Tetrahedron Lett. 2007, 48, 8878.

[11] (a) Wang, C. C.; Wang, Z. H.; Wang, L.; Chen, Q.; He, M. Y. Chin. J. Chem. 2016, 34, 1081.

(b) Fan, W. G.; Yang, Q.; Xu, F. S.; Li, P. X. J. Org. Chem. 2014, 79,10588 .

[12] (a) Jones, J. M.; Dupont, V. A.; Brydson, R.; Fullerton, D. J.; Nasri, N. S.; Ross, A. B.; Westwood, A. V. K. Catal. Today 2003, 81, 589. (b) Chu, H.; Wu, L. W. J. Environ. Sci. Health, Part A 1998, 33, 1119.

[13] Rostami, A.; Rostami, A.; Iranpoor, N.; Zolfigol, M. A. Tetrahedron Lett. 2016, 57, 192.

[14] (a) Wu, W.; Ding, Y. C.; Xie, P.; Tang, Q. J.; Pittman, C. U.; Zhou, A. H. Tetrahedron 2017, 73, 2151.

(b) Guo, T.; Wei, X. N.; Wang, H. Y.; Zhu, Y. L.; Zhao, Y. H.; Ma, Y. C. Org. Biomol. Chem. 2017, 15, 9455

(c) Guo, T.; Wei, X. N.; Zhu, Y. L.; Chen, H.; Han, S. L.; Ma, Y. C. Synlett 2018, 29, 1530.

(d) Ravi, C.; Reddy, N. N. K.; Pappula, V.; Samanta, S.; Adimurthy, S. J. Org. Chem. 2016, 81, 9964.

(e) Thanh Binh, N.; Ermolenko, L.; Al-Mourabit, A. Org. Lett. 2013, 15, 4218.

[15] Xiong, J.; Zhong, G. F.; Liu, Y. Y. Adv. Synth. Catal. 2019, 361, 550 .

[16] Shibahara, F.; Kanai, T.; Yamaguchi, E.; Kamei, A.; Yamauchi, T.; Murai, T. Chem. Asian J. 2014, 9, 237.

[17] Li, J.; Li, C.; Yang, S.; An, Y.; Wu, W.; Jiang, H. J. Org. Chem. 2016, 81, 7771.

[18] Ge, X.; Cheng, L.; Sun, F. L.; Liu, X. M.; Chen, X. Z.; Qian, C.; Zhou, S. D. Catal. Commun. 2019, 123, 32.

[19] Thanh Binh, N. Adv. Synth. Catal. 2017, 359, 1066.

[20] (a) Wang, S.; Zhou, H.; Song, S.; Wang, J. Chin. J. Org. Chem. 2015, 35, 85 (in Chinese).

(王硕，周宏勇，宋沙沙，王家喜，有机化学， 2015, 35, 85.)

(b) Yu, D.; Wu, M.; Hu, Q.; Wang, L.; Lv, C.; Zhang, L. J. Hazard. Mater. 2019, 367, 456.

(c) Masuda, K.; Ichitsuka, T.; Koumura, N.; Sato, K.; Kobayashi, S. Tetrahedron 2018, 74, 1705 .

[21] Bahuguna, A.; Kumar, A.; Kumar, S.; Chhabra, T.; Krishnan, V. ChemCatChem 2018, 10, 3121 .

[22] Hong, K. B.; Lee, C. W.; Yum, E. K. Tetrahedron Lett. 2004, 45, 693.

[23] Siddiki, S.; Kon, K.; Shimizu, K. Chem-Eur. J. 2013, 19, 14416.

[24] (a) Tukhani, M.; Panahi, F.; Khalafi-Nezhad, A. ACS Sustainable Chem. Eng. 2018, 6, 1456.

(b) Dong, Y.; Bi, J.; Zhu, D.; Meng, D.; Ming, S.; Guo, W.; Chen, Z.; Liu, Q.; Guo, L.; Li, T. Cellulose 2019, 26, 7355.

(c) Liu, B.; Zhou, H.; Li, Y.; Wang, J. Chin. J. Org. Chem. 2014, 34 2554 (in Chinese).

(刘兵，周宏勇，李云庆，王家喜，有机化学, 2014, 34, 2554.)

[25] Liu, X. M.; Chang, S.; Chen, X. Z.; Ge, X.; Qian, C. New J. Chem. 2018, 42, 16013

[26] Baran, T.; Mentes, A.; Arslan, H. Int. J. Biol. Macromol. 2015, 72, 94.

[27] Chen, H.-Y.; Peng, W.-T.; Lee, Y.-H.; Chang, Y.-L.; Chen, Y.-J.; Lai, Y.-C.; Jheng, N.-Y.; Chen, H.-Y. Organometallics 2013, 32, 5514.

[28] Gu, F.; Geng, J.; Li, M.; Chang, J.; Cui, Y. ACS Omega 2019, 4, 21421.

[29] Wang, Y.; Liu, Q.; Xu, M.; Shu, G.; Jiang, M.; Fei, G.; Zeng, M. J. Macromol. Sci. B 2017, 56, 670.

[30] Jiang, Y.; Wang, Y.; Han, Q.; Zhu, R.; Xiong, X. Chin. J. Org. Chem. 2014, 34, 2068 (in Chinese) 
(江云兵, 王彦龙, 韩骞, 朱荣俊, 熊兴泉, 有机化学, 2014, 34, 2068.)

[31] Frindy, S.; Kadib, A.; Lahcini, M.; Primo, A.; Garcia, H. ChemCatChem 2015, 7, 3307.

[32] Wang, L.; Li, B.; Xu, F.; Shi, X.; Feng, D.; Wei, D.; Li, Y.; Feng, Y.; Wang, Y.; Jia, D.; Zhou, Y. Biosens. Bioelectron. 2016, 79, 1.

[33] Verma, S. K.; Ghorpade, R.; Pratap, A.; Kaushik, M. P. Green Chem. 2012, 14, 326.
[34] Chen, M.; Huang, Z.-T.; Zheng, Q.-Y. Chem. Commun. 2012, 48 11686.

[35] Wang, P.; Tang, S.; Huang, P.; Lei, A. Angwe. Chem., Int. Ed. 2017, 56, 3009 .

[36] Chen, M.; Luo, Y.; Zhang, C.; Guo, L.; Wang, Q.; Wu, Y. Org. Chem. Front. 2019, 6, 116.

[37] Yadav, J. S.; Reddy, B. V. S.; Reddy, Y. J.; Praneeth, K. Synthesis 2009, 9, 1520.

(Li, L.; Fan, Y.) 